\title{
Perturbation of hydration layer in solvated proteins by external electric and electromagnetic fields: Insights from non-equilibrium molecular dynamics
}

Prithwish K. Nandi, Zdenek Futera, and Niall J. English

Citation: The Journal of Chemical Physics 145, 205101 (2016);

View online: https://doi.org/10.1063/1.4967774

View Table of Contents: http://aip.scitation.org/toc/jcp/145/20

Published by the American Institute of Physics

\section{Articles you may be interested in}

Electromagnetic-field effects on structure and dynamics of amyloidogenic peptides

The Journal of Chemical Physics 144, 085101 (2016); 10.1063/1.4941108

Spatially resolved dielectric constant of confined water and its connection to the non-local nature of bulk water

The Journal of Chemical Physics 145, 084901 (2016); 10.1063/1.4960775

Hydrogen bond perturbation in hen egg white lysozyme by external electromagnetic fields: A nonequilibrium molecular dynamics study

The Journal of Chemical Physics 133, 235102 (2010); 10.1063/1.3518975

Nonequilibrium molecular dynamics study of electric and low-frequency microwave fields on hen egg white lysozyme

The Journal of Chemical Physics 131, 035106 (2009); 10.1063/1.3184794

Oscillating electric-field effects on adsorbed-water at rutile- and anatase- $\mathrm{TiO}_{2}$ surfaces

The Journal of Chemical Physics 145, 204706 (2016); 10.1063/1.4967520

Perspective: Structure and ultrafast dynamics of biomolecular hydration shells

Structural Dynamics 4, 044018 (2017); 10.1063/1.4981019

\section{AIP| The Jounal of Chemical Physics}

PERSPECTIVES 


\title{
Perturbation of hydration layer in solvated proteins by external electric and electromagnetic fields: Insights from non-equilibrium molecular dynamics
}

\author{
Prithwish K. Nandi, ${ }^{a}$ ) Zdenek Futera, and Niall J. Englisha) \\ School of Chemical and Bioprocess Engineering, University College Dublin, Belfield, Dublin 4, Ireland
}

(Received 13 July 2016; accepted 31 October 2016; published online 22 November 2016)

\begin{abstract}
Given the fundamental role of water in governing the biochemistry of enzymes, and in regulating their wider biological activity (e.g., by local water concentration surrounding biomolecules), the influence of extraneous electric and electromagnetic $(\mathrm{e} / \mathrm{m})$ fields thereon is of central relevance to biophysics and, more widely, biology. With the increase in levels of local and atmospheric microwave-frequency radiation present in modern life, as well as other electric-field exposure, the impact upon hydration-water layers surrounding proteins, and biomolecules generally, becomes a particularly pertinent issue. Here, we present a (non-equilibrium) molecular-dynamics-simulation study on a model protein (hen eggwhite lysozyme) hydrated in water, in which we determine, inter alia, translational self-diffusivities for both hen egg-white lysozyme and its hydration layer together with relaxation dynamics of the hydrogen-bond network between the protein and its hydration-layer water molecules on a residueper-residue basis. Crucially, we perform this analysis both above and below the dynamical-transition temperature (at $\sim 220 \mathrm{~K}$ ), at 300 and $200 \mathrm{~K}$, respectively, and we compare the effects of external static-electric and e/m fields with linear-response-régime (r.m.s.) intensities of $0.02 \mathrm{~V} / \AA ̊$. It was found that the translational self-diffusivity of hen egg-white lysozyme and its hydration-water layer are increased substantially in static fields, primarily due to the induced electrophoretic motion, whilst the water-protein hydrogen-bond-network-rearrangement kinetics can also undergo rather striking accelerations, primarily due to the enhancement of a larger-amplitude local translational and rotational motion by charged and dipolar residues, which serves to promote hydrogen-bond breakage and re-formation kinetics. These external-field effects are particularly evident at $200 \mathrm{~K}$, where they serve to induce the protein- and solvation-layer-response effects redolent of dynamical transition at a lower temperature $(\sim 200 \mathrm{~K})$ vis-à-vis the zero-field case $(\sim 220 \mathrm{~K})$. Published by AIP Publishing. [http://dx.doi.org/10.1063/1.4967774]
\end{abstract}

\section{INTRODUCTION}

Although the thermal effects of electric and electromagnetic $(\mathrm{e} / \mathrm{m})$ fields on biological macromolecules manifest themselves in the form of heat generation, the precise mode of action of non-thermal effects is relatively less explored. ${ }^{1}$ Such fields can affect the structural and functional stability of a protein by altering its conformation through exciting its vibrational modes, as well as can induce reversible changes in its activity. ${ }^{2}$ Understanding non-thermal effects of e/m field in microwave and far-infrared regions has been the subject of extensive study, especially in relation to its possible effects on human health, ${ }^{3}$ as well as in exploring its potential applications in protein engineering and medicine. ${ }^{4,5}$

In addition to an existing experimental community working on electric- and e/m-field effects of protein denaturation and stability, ${ }^{6-8}$ efforts have also been made in recent years to build molecular-level understanding to external-field exposure by means of modern computational techniques,

\footnotetext{
a) Authors to whom correspondence should be addressed. Electronic addresses: niall.english@ucd.ie and prithwish.nandi@ucd.ie
}

such as (non-equilibrium) molecular simulation. ${ }^{9,10}$ In our previous investigation, ${ }^{11,12}$ taking hen egg-white lysozyme (HEWL) as a test case and employing the molecular dynamics method, we showed that the secondary protein structures are markedly perturbed by intense fields at $0.05 \mathrm{~V} \AA^{-1}$ (rms) (up to $\left.0.15 \mathrm{~V} \AA^{-1}\right)$, for both static oscillating fields $(2.45-500 \mathrm{GHz})$, leading to accelerated incipient denaturation. ${ }^{12}$ Further, by probing perturbations in an intra-protein hydrogen bonding network by e/m fields, we pointed out the microscopic origin of secondary structure alteration in proteins. ${ }^{13}$ It was shown that the localised translational motion of formally charged residues led to a greater disruption of associated hydrogen bonds, while the rotational motions associated with the dipolar residues led to a degree of hydrogen bonding perturbations. ${ }^{13}$

It is important to mention here that the observed biological activities of a protein ${ }^{14}$ are considered to be induced by a thin shell of water molecules adhered to the protein's outer surface-known as the hydration layer. The temperature regime within which such biochemical activities ${ }^{15-20}$ are first observed in proteins is similar to its so-called glass transition temperature, which is defined as the temperature at which the protein molecules start to exhibit large-amplitude motion. Though (rightly) debated, ${ }^{21-28}$ it is generally accepted 
that the hydration layer plays an important role in stimulating such enhanced thermal motions in protein near its glass transition point, ${ }^{23}$ as reducing the degree of hydration below a certain level significantly suppresses both the dynamical transition and the biochemical activities of a protein. An insightful recent study also emphasises that a large amplitude of thermal motions of a protein is indeed correlated with the translational motion of water molecules residing in the hydration layer. ${ }^{22}$ Water molecules in this layer form an active water-protein hydrogen-bond network which can be thought to play a key role in setting a dynamical correlation between the two. ${ }^{29} \mathrm{~A}$ few studies in the literature discuss hydrogen-bond kinetics near interfaces ${ }^{30-33}$ and the response of hydration water to external electric field. ${ }^{34-37}$ To understand the effects of staticelectric and e/m fields on a protein's biochemical activity, it is, therefore, of central relevance to explore the effect of such fields on its hydration layer. Although Ref. 12 commented briefly on dipolar orientations of water in the immediate solvation layer of HEWL, and Ref. 38 on hydration-layer water molecules' dipolar-orientation kinetics around amyloid fibrils in e/m fields, no study to date has scrutinised electric- and $\mathrm{e} / \mathrm{m}$-field effects on a protein's hydration layer in any depth, and considered potential implications for wider protein-water behaviour (such as dynamical crossover or activating protein activity).

In this work, therefore, we aim to address this important lacuna in the literature by probing perturbations in waterprotein hydrogen bond dynamics and translational diffusion of both the protein and its hydration layer. Using HEWL as a test case, we calculate the statistical distributions of lifetime of each water-protein hydrogen bond in between (re-)formation and breakage, under zero-field conditions and in the presence of static-electric and e/m fields. The effects of e/m fields on the translational self-diffusivity of the protein and its hydration layer are also explored here, as well as a range of other protein-water properties.

\section{METHODOLOGY}

All non-equilibrium MD simulations were performed, using GROMACS version 5.1.2, ${ }^{39}$ in a similar way to Refs. 12 and 13; a brief account is given here for the sake of completeness. The OPLS ${ }^{40,41}$ and $S P C / E^{42}$ potential models were used for HEWL and water, respectively. Triclinic wild-type HEWL, namely, $2 \mathrm{LZT}^{43}$ is a globular protein with molecular mass $14320 \mathrm{Da}$ and an overall charge +8 e appropriate to $\mathrm{pH}$ 7. A corresponding number of $\mathrm{Cl}$ counter-ions were added to the solution to make the whole system electroneutral. The protein was placed at the centre of a cubic periodic box with $(x, y, z)$ dimensions of $\sim 70 \AA$, respectively, in the laboratory Cartesian frame of the original structure, with $\sim 10000$ molecules of SPC/E water surrounding the protein structure. The SPC/E water model was used due to the compatibility of OPLS with SPC-type models ${ }^{40,41}$ and its superior estimate for both self-diffusivity and freezing point than SPC water. ${ }^{42}$ In this study, the e/m fields were applied along the laboratory z-axis only, considering negligible difference of results when electric and e/m fields were applied to wild-type HEWL and its mutants along each Cartesian axis with respect to a laboratory frame. Each simulation was carried out at constant temperature and constant pressure. Temperature control was imposed using a Nosé-Hoover thermostat and a pressure of 1 bar was maintained throughout the entire simulation using the Parrinello-Rahman barostat. Holonomic constraints were handled by the LINCS $^{44}$ method. The smooth particle-mesh Ewald (SPME) method ${ }^{45}$ was used to handle the long-range electrostatic interactions. Each system was simulated at two different temperatures (200 and $300 \mathrm{~K}$ )—so as to be above the putative dynamical transition temperature of $\sim 220 \mathrm{~K}$ for HEWL. ${ }^{29}$ For each simulation, the system was equilibrated for a total time of $20 \mathrm{~ns}$, and, once the system was thermally stabilised, production run of 40 and 20 ns were performed at 200 and $300 \mathrm{~K}$, respectively.

Uniform e/m fields were applied with the electric component $\mathbf{E}$ acting along the laboratory $z$-direction $\mathbf{E}(\mathrm{t})$ $=E_{\max } \cos (\omega \mathrm{t}) \mathbf{k}$ as described in previous studies, ${ }^{11,12,46,47}$

$$
m_{i} \ddot{r}_{i}=f_{i}+q_{i} E(t),
$$

where $q_{i}$ denotes the charge and $f_{i}$ the force on site $i$ due to the intermolecular potential. A static field was also applied. ${ }^{12}$ Classical mechanics was used for the treatment of the $\mathrm{e} / \mathrm{m}$ absorption, since the experimental spectrum of liquid water is continuous in the low-frequency microwave region. ${ }^{48}$ The applied e/m fields were of frequency $\vartheta=2.45-100 \mathrm{GHz}$ $\left(0.082-3.3 \mathrm{~cm}^{-1}\right.$ ) and of r.m.s. intensity $E_{r m s}=0.02 \mathrm{~V} / \AA$ (and also $0.02 \mathrm{~V} / \mathrm{A}$ in the static case), with $2.45 \mathrm{GHz}$ being used widely for industrial and civilian use in the microwave region. ${ }^{4}$ Although this field intensity is some three orders of magnitude larger than those applied typically in industry, ${ }^{4}$ it has been found from the equilibrium MD simulation that electric field intensities in condensed water phases are in the range of around 1.5 to $2.5 \mathrm{~V} / \AA{ }^{47}$ giving rise to de facto "signal-to-noise" ratios of between 75 and 125:1 for the intrinsic to applied fields in the present work. In our previous work, ${ }^{12}$ an r.m.s. intensity of $0.01 \mathrm{~V} / \AA ̊ \AA$ led to statistically indistinguishable changes in the HEWL mutants' RMSD's or gross dipolar alignments over 25 ns vis-à-vis zero-field conditions in either static or 2.45 $\mathrm{GHz}$ e/m fields; this observation is in accord with the gasphase simulations of polyalinines in static $0.01 \mathrm{~V} / \AA$ electric fields performed by Calvo and Dugourd. ${ }^{49}$ Indeed, previous analysis has indicated that r.m.s. intensity exhibits a lineartype response to dipole alignment, a behavior we would also expect in the current analysis. The external fields were applied in conjunction with the NPT coupling and are referred to as non-equilibrium NPT (NNPT) simulations. ${ }^{47}$ These simulations were performed in order to isolate the athermal effects as much as possible from the thermal effects. A series of NNPT simulations were carried out at both 200 and $300 \mathrm{~K}$ and 1 bar for 40 and $20 \mathrm{~ns}$ in static, 2.45, 20, and $100 \mathrm{GHz}$ fields at 200 and $300 \mathrm{~K}$, respectively, as well as equilibrium, zero-field simulations (corresponding to around 100 and 50 full cycles for the lowest-frequency $2.45 \mathrm{GHz}$ fields at 200 and $300 \mathrm{~K}$, respectively).

To identify hydrogen bonds between the protein atoms and the water molecules at each snapshot over the entire simulation time, the geometric criteria as described by Durrant and $\mathrm{McCammon}^{50}$ were adopted. Only oxygen, nitrogen, fluorine, or sulphur atoms were considered as the heavy-atom 
participants in forming hydrogen bonds. The distance between the donor and the acceptor heavy atoms must be less than 3.5 $\AA$ and the angle between the hydrogen atom, the donor heavy atom, and the acceptor heavy atom must be less than $30^{\circ}$. For each detected hydrogen bond, we calculated the lifetime defined as the percentage of time it appears over the entire simulation time. During its entire occupation time, a hydrogen bond can disappear and re-appear again for which we describe as "breakage" and "formation" of a bond, respectively. The time of existence between such a subsequent "formation" and "breakage" event is defined as the lifetime of a bond. ${ }^{13}$ For each lifetime, we have associated two unique quantities, namely, "distribution of lifetime" and "partial lifetime contribution." The "distribution of lifetime" of a lifetime is defined as the number of its occurrences (in percentage terms) over the entire simulation time whereas the "partial lifetime contribution" defines the proportion of time (also as a percentage) it contributes to the occupation time of that particular hydrogen bond.

The translational self-diffusion coefficient was estimated from the MSD in long time limit using the following Einstein relationship: ${ }^{47}$

$$
D_{T}=\lim _{t \rightarrow \infty} \frac{1}{6 t}\left\langle\left|\boldsymbol{r}\left(t_{0}+t\right)-\boldsymbol{r}\left(t_{0}\right)\right|^{2}\right\rangle .
$$

Here, $\mathrm{D}_{\mathrm{T}}$ is the diffusion coefficient, $\mathbf{r}\left(\mathrm{t}_{0}+\mathrm{t}\right)$ and $\mathbf{r}\left(\mathrm{t}_{0}\right)$ are the position of the centre of mass of a water molecule or a protein non-hydrogen atom, at times $t$ and $t_{0}=0$, respectively. The angular bracket represents the ensemble average over both molecules and time origins. For calculating self-diffusion coefficients, the slope of the MSD curve was determined using the least-squares linear regression, and the correlation coefficient (r) was determined for each case, to ascertain the goodness of fit.

\section{RESULTS AND DISCUSSIONS}

The primary focus of this study is to explore the electricand $\mathrm{e} / \mathrm{m}$-field-induced perturbation of the hydrogen-bond kinetics, i.e., the speeds at which the hydrogen bonds are broken up and re-established, of the water-protein hydrogen bond network. Another aim is to quantify the changes in translational dynamics of the protein and its hydration layer, as a result of the application of external fields. Therefore, proper identification of a hydration layer is essential. Water molecules surrounding a protein have three broadly distinct categories: ${ }^{21}$ those confined in deep clefts of the folded protein known as "bound water," whereas those interacting directly with the exposed-surface protein atoms constitute the hydration layerknown also as "surface water." The third category is "bulk water"-in this case, water molecules not directly in contact with the protein surface, but which exchange continuously with the surface water. Estimating the thickness of a hydration shell is a debated subject and has been studied over the past few years. Both experimental techniques like dielectric relaxations, followed by more recent Terahertz-spectroscopy methods $^{51}$ as well as computer-simulation studies, have been employed to estimate the hydration shell's thickness. While most studies of rotational friction provide an estimate of 3$4 \AA$, more recent studies show that depending on the size of

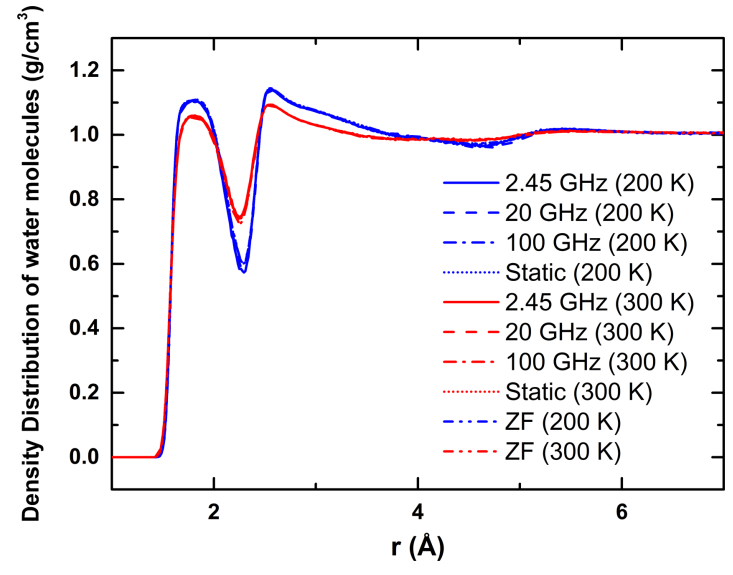

FIG. 1. Density distribution of water molecules in the surroundings of the HEWL surface for $200 \mathrm{~K}$ and $300 \mathrm{~K}$. For each temperature, five different external-field conditions are used. ZF stands for zero-field conditions, "static" implies a static field with amplitude $0.02 \mathrm{~V} / \AA$, and " $2.45 \mathrm{GHz}$," "20 GHz," and "100 GHz" represent the corresponding e/m fields with r.m.s. intensity of $0.02 \mathrm{~V} / \AA$. Two distinct solvation layers are evident for all cases. Although temperature is seen to influence the spatial density distribution, external fields do not show any discernible effects on the distribution.

a protein, it can influence water molecules residing within a thickness of more than even $10 \AA{ }^{29}$ In this study, to identify the hydration layer around HEWL, we computed the density distribution (Fig. 1) of the water molecules' centres of masses from the protein surface. Proteins, of course, do not have a regular shape. Normalising the density distribution requires accurate estimation of the hydration shells' complex-shaped volumes around the protein. Using the state-of-the-art Voronoi cell analysis, ${ }^{52}$ we computed the "Voronoi" volumes associated with each molecule in the hydration layer, thus estimating the hydration shell's total volume. The resultant density distribution of water around the protein's outer surface for two different temperatures ( $200 \mathrm{~K}$ and $300 \mathrm{~K}$ ) is shown in Fig. 1. For each temperature, the distribution is plotted for five distinct field conditions: zero field (ZF), static field with $0.02 \mathrm{~V} / \AA$ amplitude (Static), and three e/m fields with frequencies 2.45 , 20 , and $100 \mathrm{GHz}$ and r.m.s. intensity $E_{r m s}=0.02 \mathrm{~V} / \AA$. Two distinct hydration layers around the protein surface are very much evident for each case studied here. While at $200 \mathrm{~K}$ the first and the second hydration layer extends up to a distance of $\sim 2.3 \AA$ and $\sim 4.6 \AA$, respectively, for $300 \mathrm{~K}$, though the first minima are located at $\sim 2.25 \AA$, the location of the second minima is not well defined. In both cases, the density approaches to the bulk value at about $6 \AA$. However, it is interesting to notice from Fig. 1 that the application of static and oscillating electric field has negligible impact on the shape of this time-averaged density distribution. Water molecules interact with the polar ionic groups at the protein surface- the nature of such an interaction is Coulombic. This charge-dipole interaction falls of as $r^{-453}$ in the second solvation layer; this interaction is weakened substantially and water-water interactions dominate in this layer. Thus, water molecules in the second layer-no longer in direct contact with the protein surface-have higher mobility relative to those in the first one, with properties approaching those of the bulk. So, in practice, the first monolayer where a large proportion of water molecules remain spatially restricted or coordinated with the protein can be effectively regarded as the 

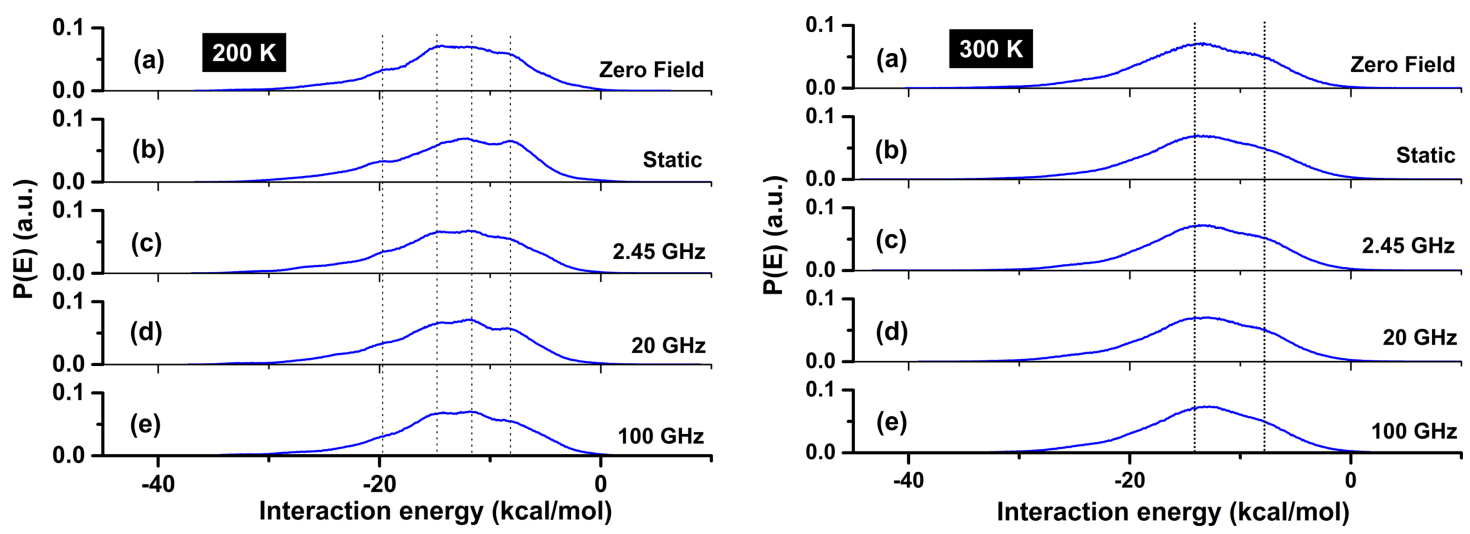

FIG. 2. Distribution of interaction energy of water molecules present in the first solvation layer around the HEWL surface, with HEWL itself (with no energyevaluation cutoff applied). A prominent presence of distinct features in the above distribution is seen for $200 \mathrm{~K}$ (on the left). As temperature increases, such features disappear gradually. The presence of external electric fields influence the distribution to some extent for $200 \mathrm{~K}$, though at higher temperature (300 K, on the right), the external field influences this distribution to a lesser extent.

hydration shell of the protein. The spatial distribution and orientation of water molecules in this hydration shell are expected to differ from the bulk as the local coordination of these water molecules would be very different depending on the field of interaction exerted by the protein. It is quite well-known that near the hydrophobic protein residues, water molecules direct its hydrogen bonding sites away from the surface, while the reverse orientation could be realised near the hydrophilic residues. ${ }^{29}$ Indeed, the probability distribution of interaction energy of the hydration water molecules with the protein can be considered to be a reasonable metric for the quantitative representation of molecular orientation in the hydration shell. Here, the interaction energy of each water molecule present in the first hydration layer around HEWL with the protein itself was computed (with no energy-evaluation cutoff applied to the van der Waals and unshielded Coulombic interactions), and its probability distribution determined. We have plotted this in Fig. 2, where the left panel and the right panel show the results for $200 \mathrm{~K}$ and $300 \mathrm{~K}$, respectively. Five rows from (a) to (e) represent data for zero-field, static field, and $\mathrm{e} / \mathrm{m}$ fields $(2.45,20$, and $100 \mathrm{GHz})$, respectively. Interestingly, the distribution at lower temperature, i.e., $200 \mathrm{~K}$, for a zero-field case, exhibits well-defined structures, indicating the presence of groups of water molecules with unique orientations. Such sharply defined structures almost fade out near room temperature $(300 \mathrm{~K})$. One might argue that thermal fluctuations at $300 \mathrm{~K}$ are sufficient enough to disrupt ordered molecular arrangements as seen at $200 \mathrm{~K}$, which is well below the so-called protein glass transition temperature $(220 \mathrm{~K})$ where the molecular motion remains is almost arrested (vide infra). At $200 \mathrm{~K}$, this structured distribution is altered most by the presence of the static field where a well-defined peak is seen to emerge at $\sim 2 \mathrm{kcal} / \mathrm{mol}$ and the "hump" which was present originally at a position of $\sim-10 \mathrm{kcal} / \mathrm{mol}$ for the zero-field case is seen to disappear. In e/m fields, the results are almost comparable with the zero-field case. On the other hand, for $300 \mathrm{~K}$, the effect of electric field on dictating molecular orientation in the hydration layer is largely negligible. This mirrors the findings of Ref. 47, in which the self-diffusivity is enhanced more by the external electric fields in supercooled water relative to room-temperature liquid water: the external field allows for more substantial crossing of free-energy barriers for local water re-orientation at a lower temperature.

As mentioned previously, it is believed widely that the hydration layer adopts a decisive role in activating the bio-functionality of a biomolecule. Large-amplitude thermal motion of protein molecules is often conjectured to be correlated with the mobility of the water molecules present in the hydration layer. ${ }^{22}$ Diffusivity is a quantitative measure of a particle's thermal motion. Here, we present a rigorous computation of the translational self-diffusivities $\left(D_{T}\right)$ for both the protein and the associated hydration layer using the Einstein relationship (cf. Sec. II), and we also investigate the effects of external field in manipulating the self-diffusivities of the protein and its hydration layer.

For computing MSDs and diffusivity of the hydration layer, a rigorous filtration process was followed throughout the independent subsections of the entire trajectories (20-40 ns). The centres of mass of water molecules were tracked, and those of them that remained in the first solvation layer for at least $90 \%$ of each subsection were utilized to compute the MSD of the hydration layer. The MSDs could then be averaged over all subsections. When applied to HEWL, only the coordinates of all non-hydrogen atoms were taken into account for computing MSD and $\mathrm{D}_{\mathrm{T}}$ (cf. Sec. II). MSD plots of HEWL and the hydration layer are shown in Figs. S1 and S2 (cf. the supplementary material). Self-diffusivity values of both the protein and the hydration layer at 200 and $300 \mathrm{~K}$ under various external field conditions are shown in Figs. 3(a) and 3(b), respectively. Experimental values of self-diffusivity of HEWL and bulk water are available in the literature near room temperature. From both the $1 \mathrm{H}$ NMR-PFG ${ }^{54}$ and the diaphragm cell technique, ${ }^{55,56}$ the bulk diffusivity value of water at $298 \mathrm{~K}$ is estimated to be approximately $2.3 \times 10^{-9} \mathrm{~m}^{2} / \mathrm{s}$ which is almost an order greater than the $\mathrm{D}_{\mathrm{T}}$ value of the hydration layer at $300 \mathrm{~K}$, as estimated in the current study, indicating a striking difference of dynamical features between themhydration water being viewed as a relatively confined layer of surface water. A reasonable agreement exists between current MD data of $\mathrm{D}_{\mathrm{T}}\left(\sim 0.087 \times 10^{-9} \mathrm{~m}^{2} / \mathrm{s}\right)$ for HEWL (ZF 

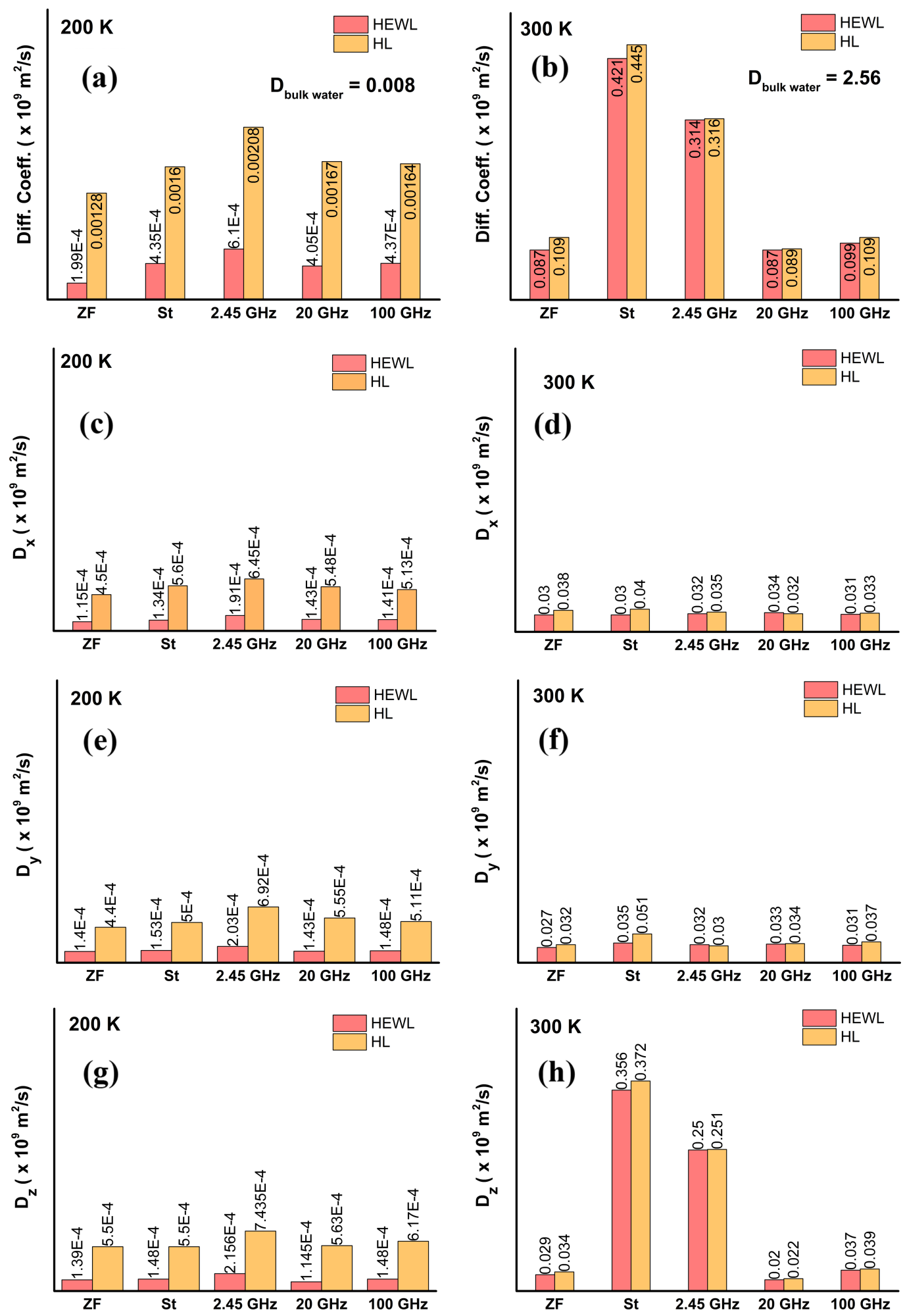

FIG. 3. Translational self-diffusion constant of the protein (HEWL) and its hydration layer (HL) at five different field conditions for $200 \mathrm{~K}$ (a) and $300 \mathrm{~K}$ (b). It is interesting to note that the external electric field influences the translational diffusivity of both the protein and the hydration layer considerably, though for $300 \mathrm{~K}$, for higher frequency fields $(20 \mathrm{GHz}$ and $100 \mathrm{GHz}$ ), this effect is not seen. To explore the electrophoresis effect, the self-diffusivity values are plotted ((c)-(h)) along the parallel (z-direction) and perpendicular directions ( $\mathrm{x}$ - and $\mathrm{y}$-directions) of the applied external electric field.

case) at $300 \mathrm{~K}$ and the available experimental data (from depolarised light-scattering techniques $)^{57}$ where a value of $\left(\sim 0.106 \times 10^{-9} \mathrm{~m}^{2} / \mathrm{s}\right)$ is reported at $293 \mathrm{~K}$; this validates the correctness of our calculations. It is to be noted that accurate calculations of experimental $\mathrm{D}_{\mathrm{T}}$ are strongly affected by the presence of counter-ions and the experimental hydrodynamic data are usually corrected by the viscosity data of water at $298 \mathrm{~K}$, adding further errors in those estimations. It is quite interesting to note that the value of $\mathrm{D}_{\mathrm{T}}$ for the water present in the hydration shell at $200 \mathrm{~K}\left(1.28 \times 10^{-12} \mathrm{~m}^{2} / \mathrm{s}\right)$ 
is substantially lower than the corresponding bulk value, and emphasises their ultra-low mobility - an observation in accordance with the experiments reporting a glassy-state of hydrated proteins at this low temperature range. The HEWL protein is also seen to have an ultra-low diffusivity of $\sim 1.99 \times 10^{-13}$ $\mathrm{m}^{2} / \mathrm{s}$ (cf. Fig. 3(a)), although one order less than that of its surrounding hydration layer, at this temperature. For the case of $\mathrm{T}=300 \mathrm{~K}$, the translational mobility of the protein and its hydration layer, as reflected from their $\mathrm{D}_{\mathrm{T}}$ values, are almost coherent, implying a dynamical correlation between them.

As mentioned earlier, the biological activities of proteins are believed to be related with their large scale thermal fluctuation at or above its so-called glass transition temperature $(\sim 220 \mathrm{~K})$. It would be quite interesting (both academically and from a potential industrial perspective) to study whether one can induce such biological activities in such proteins even below the glass-transition temperature by appropriately engineering their thermal mobility by external perturbing fields (e.g., static or e/m electric fields) of suitable amplitude and frequency. We have thus estimated how the electric field can modulate the translational self-diffusivity of protein and its hydration layer when compared to the ZF conditions. While in Figs. 3(a) and 3(b), we have shown the diffusivity plots for $200 \mathrm{~K}$ and $300 \mathrm{~K}$, respectively, in Figs. 3(c)-3(h), the diffusivity values are shown for these two temperatures in directions parallel and perpendicular to the direction of the applied electric field (z-direction), in particular, to disentangle the electrophoresis effect from the enhancement of translational self-diffusivity. As shown in Fig. 3(a), at $200 \mathrm{~K}$, for both the HEWL and the hydration layer, $\mathrm{D}_{\mathrm{T}}$ is increased significantly from its $\mathrm{ZF}$ value. The increment is maximum (almost by $\sim 200 \%$ ) for the e/m field with microwave-range frequency (i.e., $2.45 \mathrm{GHz}$ ), while for other field conditions, the increment varies in between $100 \%$ and $120 \%$ approximately; HEWL electrophoresis (due to an overall HEWL charge) was retarded at $200 \mathrm{~K}$ under static-field conditions by supercooled SPC/E water with a substantially higher degree of friction than at room temperature. At $200 \mathrm{~K}$, the $2.45 \mathrm{GHz}$ and lower-frequency microwave fields are more effective at reducing the de facto friction of supercooled SPC/E water than static fields due to the enhanced dipole-rotational oscillation, ${ }^{47}$ so this serves to rationalise the boosting of the HEWL and water self-diffusivities at $200 \mathrm{~K}$ in the presence of e/m fields at $200 \mathrm{~K}$, despite the absence of gross electrophoresis of HEWL in e/m fields. For studies at room-temperature (300 K) also (cf. Fig. 3(b)), the $\mathrm{D}_{\mathrm{T}}$ value, for the static field case, increases dramatically by an amount of approximately $400 \%$. The enhanced translational self-diffusivity of HEWL itself, and that of its accompanying hydration-water layer, in static fields arises in the main from gross electrophoretic motion, ${ }^{12}$ with the de facto Reynolds number of the protein indicating induced laminar flow at both 200 and $300 \mathrm{~K}$ from Stokes' law considerations. ${ }^{12}$ There was relatively little "break-up" of the hydration-layer, with few such water molecules left in HEWL's wake, owing to these laminar-flow conditions. ${ }^{12}$ In rather stark contrast, for e/m fields at $300 \mathrm{~K}$, the increment is pronounced only for microwave-frequency $(2.45 \mathrm{GHz})$ where an increment of approximately $250 \%$ from the $\mathrm{ZF}$-value is observed. As the frequency is increased, the perturbation of the values of translational self-diffusivity is seen to essentially disappear as compared to the ZF-case. This mirrors the findings of Ref. 47, in which water selfdiffusivity is enhanced more by external electric fields in supercooled water relative to room-temperature liquid water, and higher-frequency external fields converged towards the zero-field results: more crossings of the (local) free-energy rotational landscape occur at a lower temperature due to an external field, in addition to external fields becoming so rapidly varying with few-picosecond direction-reversal time scales (i.e., half-periods) that there is insufficient time for appreciable water re-orientation to "track" the fast-oscillating external field. Noticeably, the external electric field perturbs the translational self-diffusivity for both the HEWL and its hydration layer by a similar extent. Thus, the dynamical correlation between the protein and its hydration layer which is observed for the $\mathrm{ZF}$ condition is seen to be maintained even in the presence of external perturbing fields, emphasising the intrinsic nature of this dynamical correlation. At this stage, it is interesting to explore the key mechanistic considerations that can explain such an observed dynamical behaviour in a hydrated protein in the presence and absence of external electric and e/m fields.

As mentioned earlier, hydration-layer water is fundamentally different from bulk water. This arises primarily from the characteristically different protein-water hydrogen bond network in the vicinity of the protein's outer surface as compared to the hydrogen-bond network that water molecules experience in the bulk. From previously outlined simple mechanistic considerations, ${ }^{12}$ one may expect that charged residues (positive or negative) will undergo localised field-induced translational motion, thus serving to perturb hydrogen bonds in which their constituent atoms participate (either as donors or acceptors). It is also expected that residues with significant dipoles would undergo local rotational motion, which would also lead to accelerated break-up of hydrogen bonds (and possible re-formation, although not guaranteed), thereby

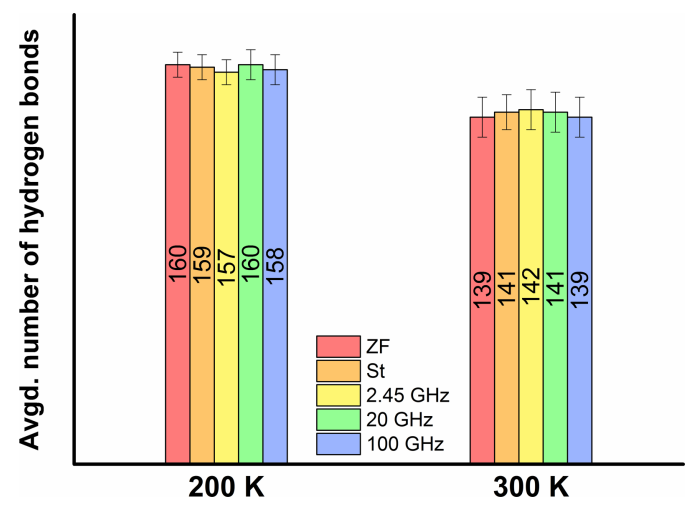

FIG. 4. Time-averaged number of hydrogen bonds that exist between the protein surface and its hydration layer at any instant for two different temperatures $(200 \mathrm{~K}$ and $300 \mathrm{~K})$. For each temperature, results are shown for the five different field conditions. It is interesting to note that though the temperature has a significant effect on influencing the number, the external electric fields do not have much effect on the average value of protein-water hydrogen bonds. The numbers in the boxes show the average value of hydrogen bonds at that field condition. 

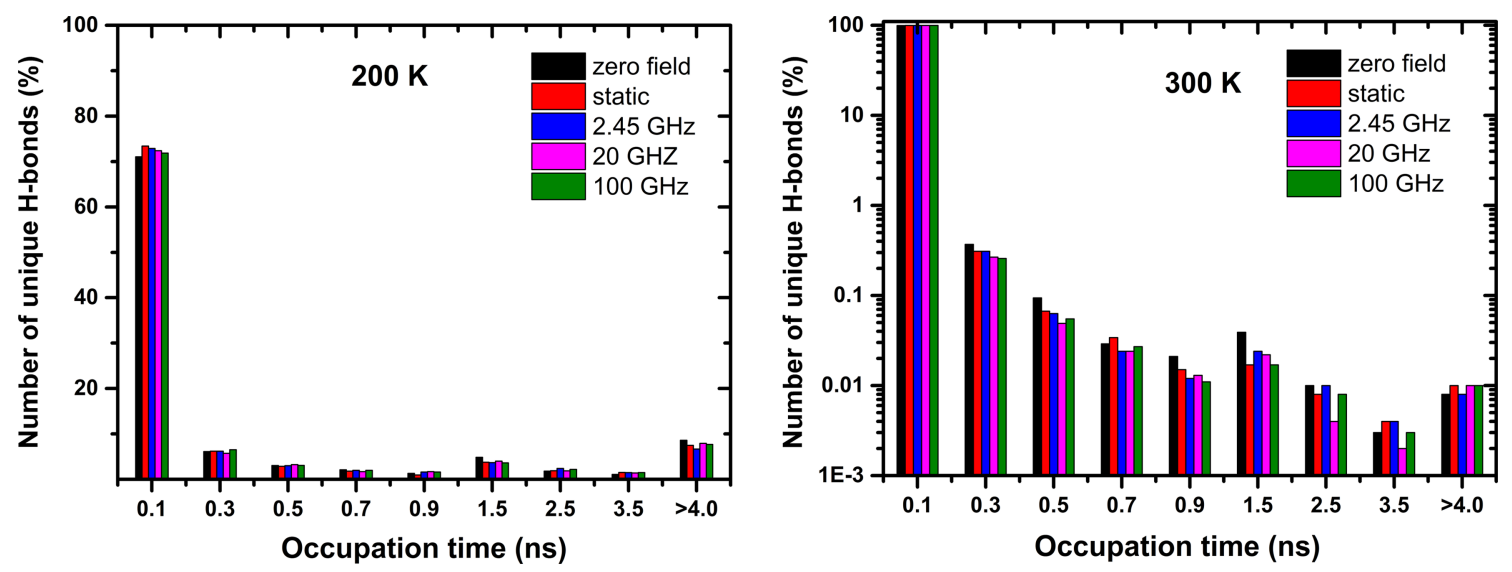

FIG. 5. Histogram showing the number of unique water-protein hydrogen bonds with an occupation time (shown along the abscissa) for $200 \mathrm{~K}$ (left panel) and $300 \mathrm{~K}$ (right panel). While for $200 \mathrm{~K}$, H-bonds with occupation time $0.1 \mathrm{~ns}$ or less is about $70 \%$ of the total bonds appeared during the entire simulation, for $300 \mathrm{~K}$, almost $99.5 \%$ bonds having lifetime less than $0.1 \mathrm{~ns}$. This indicates that as T is increased the average lifetime of the H-bonds decreases. For $200 \mathrm{~K}$, the application of external electric field increases the number (in \%) for bonds with lifetime less than 0.1 ns by $\sim 2.5 \%, \sim 1.9 \%, \sim 1.4 \%$, and $\sim 0.8 \%$ for static, 2.45 $\mathrm{GHz}, 20 \mathrm{GHz}$, and $100 \mathrm{GHz}$, respectively, indicating the reduction of average lifetime of H-bonds in the presence of external electric field. For $300 \mathrm{~K}$ also a similar trend exists though the magnitude of increment is less. At $300 \mathrm{~K}$, the ordinate axis is shown on a logarithmic scale to highlight the changes for hydrogen bonds with lifetime of more than $0.1 \mathrm{~ns}$.

lowering lifetimes (or "occupation times") of these hydrogen bonds very substantially. Similarly, water molecules, with a large ratio of permanent dipole moment vis-à-vis moment of inertia, ${ }^{12,58,59}$ also exhibit substantial rotational motion in the presence of external electric fields, giving rise to changes in hydrogen-bond dynamics; in the context of protein-hydration
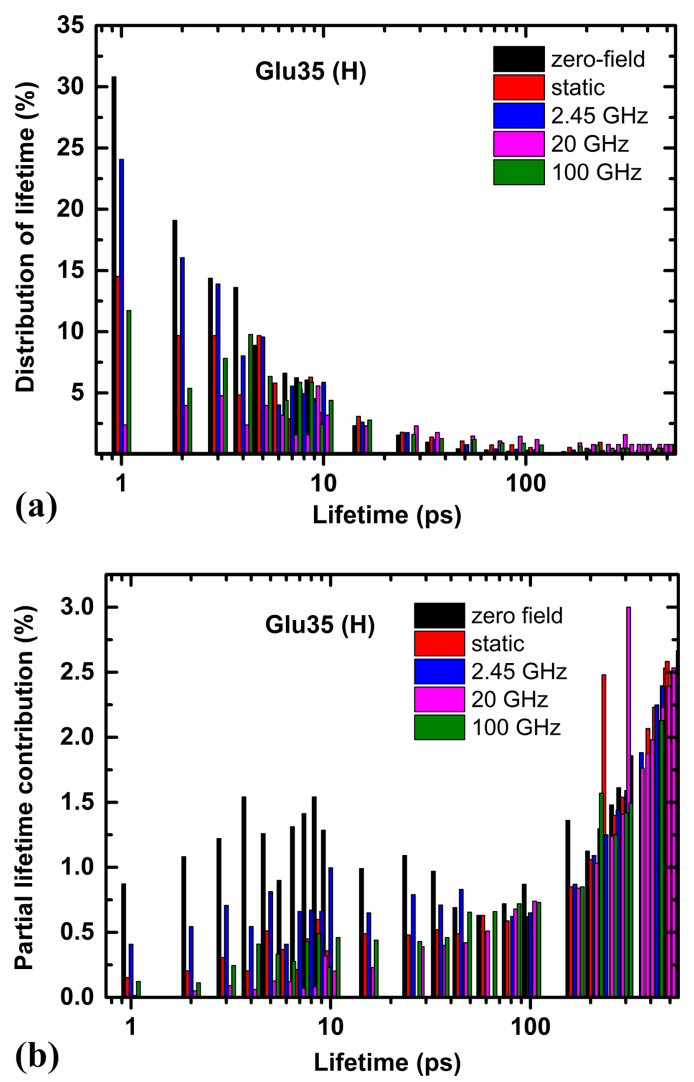

FIG. 6. Plots showing distribution of lifetime (a) and partial lifetime contribution (b) of the lifetime for the hydrogen bond formed by a Glu35 proton with a water oxygen atom, at $300 \mathrm{~K}$. water, such effects exhibit substantial local variation, depending on the interactions with specific local residues and surface topology. In previous studies of water in e/m fields, ${ }^{46,47}$ it was shown that field-induced dipolar rotational motion of water molecules led to an increase in hydrogen-bond kinetics (and self-diffusivity via roto-translational coupling), ${ }^{60-63}$ i.e., the
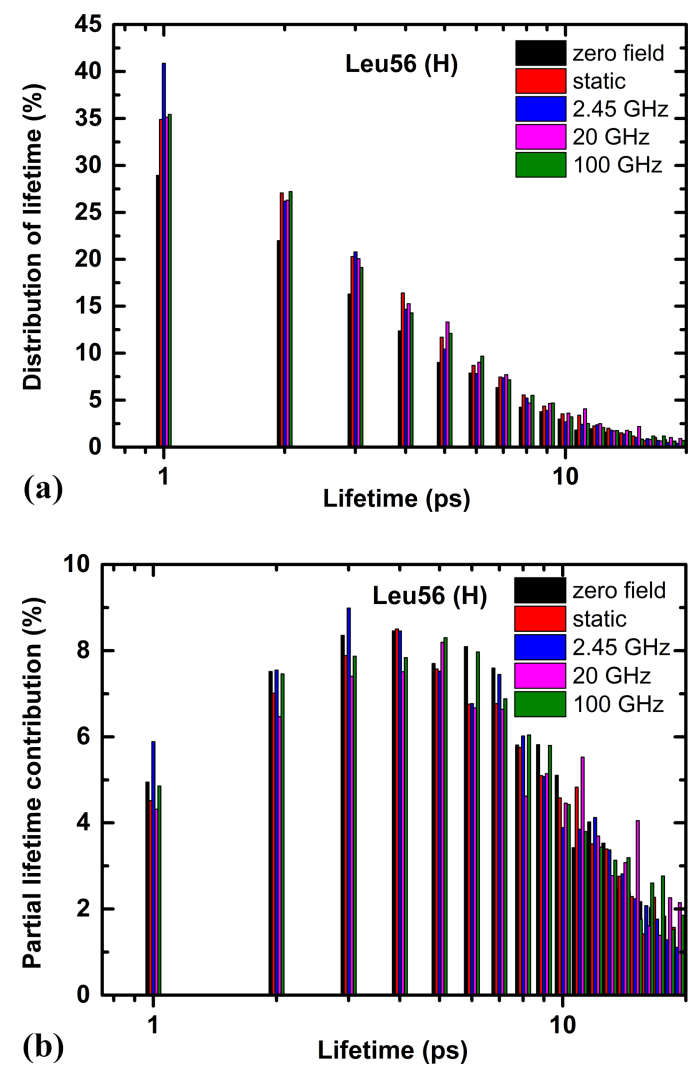

FIG. 7. Plots showing distribution of lifetime (a) and partial lifetime contribution (b) of the lifetime for the hydrogen bond formed by a Leu56 proton with a water oxygen atom, at $300 \mathrm{~K}$. 
speeds at which the hydrogen bonds were broken up and reestablished. In the following discussion, therefore, we shall highlight the key features of the effects of electric fields on the protein-water hydrogen bond dynamics.

Using the geometric criteria mentioned in Sec. II, the number of existing hydrogen bonds between the protein and the hydration layer was estimated every 1 ps for a total duration of $20 \mathrm{~ns}$. In our analysis, we tagged each hydrogen bond with a unique bond identification number (UBIN) decided by the atom-tag of the acceptor and the receptor. For each run, we counted how many such UBINs are recorded. As temperature is increased from 200 to $300 \mathrm{~K}$, the net UBIN increased by a factor of approximately 75, indicating the high mobility of both the protein structural residues and the water molecules in the hydration layer (also supported by the self-diffusivity data shown in Figs. 3(a) and 3(b)): plainly, this results in a more extensive "exposure" to various possible orientations which satisfy the geometric criteria of protein-water hydrogen-bond formation. In addition, for each temperature, the application of external electric field also enhances this UBIN by a significant proportion from its ZF-value. Although at $200 \mathrm{~K}$, depending on the external-field condition, the net increment in UBIN from the ZF-value is by $8 \%-22 \%$ (cf. Table S3 of the supplementary material), for $300 \mathrm{~K}$, the net increment is 6\%-10\% (cf. Table S3). This increment is also due to the enhanced number of possible molecular arrangements and configurations induced by the external electric field on charged or dipolar protein residues, and also strongly dipolar water molecules. Most of these hydrogen bonds are short-lived, having a total
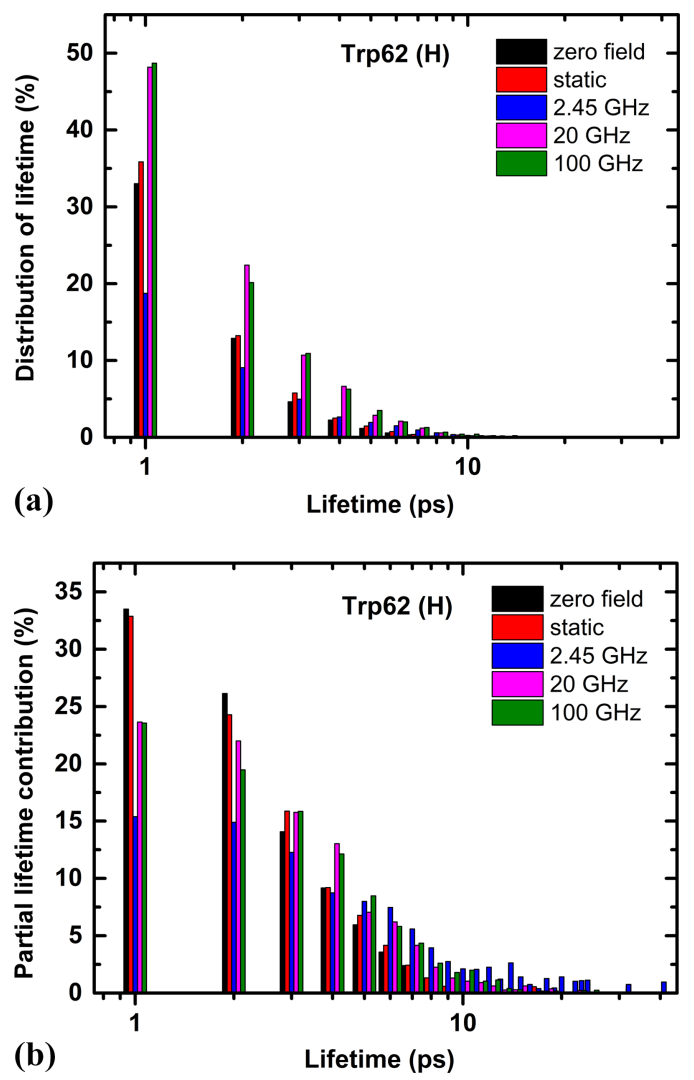

FIG. 8. Plots showing distribution of lifetime (a) and partial lifetime contribution (b) of the lifetime for the hydrogen bond formed by a Trp62 proton with a water oxygen atom, at $300 \mathrm{~K}$. aggregated-lifetime less than $20 \%$ of the entire simulation time (20 ns) (cf. Fig. 5). In Tables S1 and S2 (cf. the supplementary material), a comprehensive list of 129 protein-residues is given, where we have marked boxes corresponding to those residues having the aggregated-lifetime exceeding $20 \%$ of the entire simulation time. The number specified in Tables $\mathrm{S} 1$ and S2 corresponds to the number of active hydrogenbond-forming-sites of each residue. Thus, these tables serve to represent an overall picture of hydrophilicity of HEWL under various external field conditions. For the zero-field case, at $200 \mathrm{~K}$, almost $57 \%$ of the 129 residues in HEWL protein form hydrogen bonds with water having aggregated-lifetime more than $20 \%$, and for $300 \mathrm{~K}$, this number reduces to only $7 \%$, indicating that the thermal fluctuations at $300 \mathrm{~K}$ are sufficient to rupture hydrogen bonds for most of the residues which form long-lived hydrogen bonds with water at $200 \mathrm{~K}$ (cf. Tables S1 and S2 of the supplementary material)_in some cases, with little scope for re-formation. The application of external electric fields promotes the number of residues forming long-lived hydrogen bonds at $300 \mathrm{~K}$ to almost $12 \%$, except in the case of microwave frequency $(2.45 \mathrm{GHz})$, where a marginal increase is observed. In Fig. 4, we have shown the time-averaged value of the number of hydrogen bonds existing at an instant between the protein and the water molecules present in the hydration layer, for the zero-field case and for the external-field conditions studied. The numbers shown in the boxes of the bar-plots represent the actual number of protein-water hydrogen bonds. The standard deviation is also shown in the plot as error bars. It is clear that application of external electric fields results in
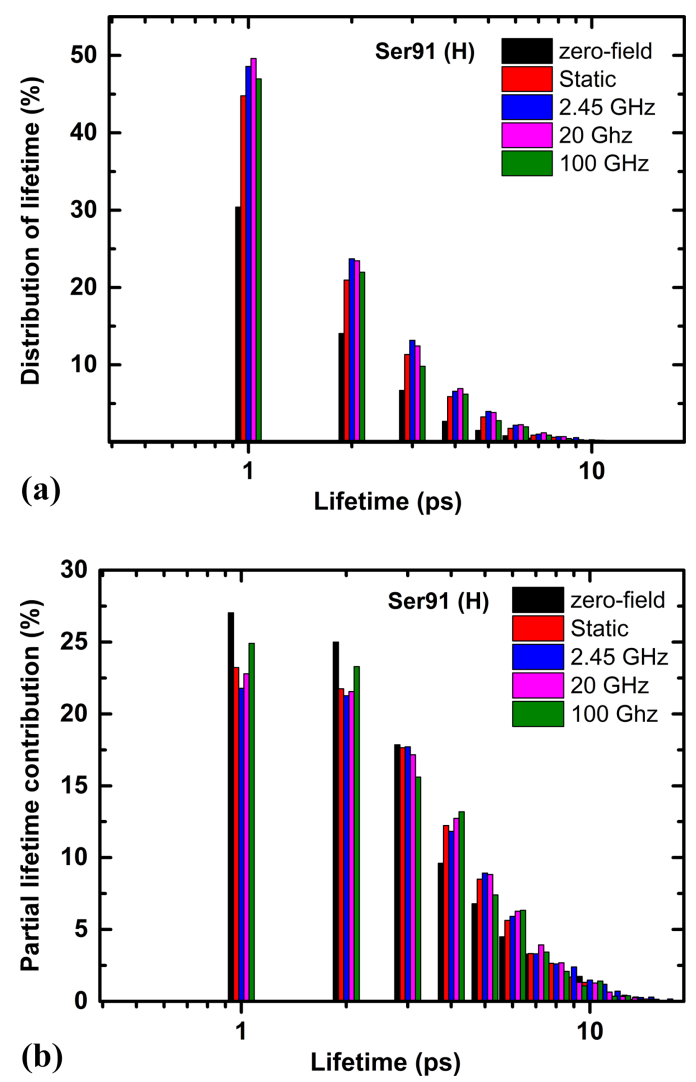

FIG. 9. Plots showing distribution of lifetime (a) and partial lifetime contribution (b) of the lifetime for the hydrogen bond formed by a Ser91 proton with a water oxygen atom, at $300 \mathrm{~K}$. 
little increase in the net number of hydrogen bonds per se. Their kinetics, as opposed to raw number, however, is a markedly different matter. Fig. 5 depicts the histogram showing number of unique water-protein hydrogen bonds with an occupation time at 200 and $300 \mathrm{~K}$. Although at $200 \mathrm{~K}$, hydrogen bonds with lifetime $0.1 \mathrm{~ns}$ or less account for about $70 \%$ of the total hydrogen bonds appearing during the entire simulation, for $300 \mathrm{~K}$, almost $99.5 \%$ of bonds have lifetime less than $0.1 \mathrm{~ns}$. This indicates, as temperature is increased, that the average lifetime of the hydrogen bonds decreases. At $200 \mathrm{~K}$, the application of external electric field increases the proportion of hydrogen bonds with lifetimes of $0.1 \mathrm{~ns}$ (or less) by $\sim 2.5 \%, \sim 1.9 \%, \sim 1.4 \%$, and $\sim 0.8 \%$ under static, 2.45 $\mathrm{GHz}, 20 \mathrm{GHz}$, and $100 \mathrm{GHz}$ fields, respectively, indicating a reduction of the average lifetime of hydrogen bonds in the presence of external electric fields. At $300 \mathrm{~K}$, a similar trend holds, although the magnitude of increment is less dramatic. In general, most quickening vis-à-vis the zero-field case was observed for hydrogen bonds formed between water protons and electronegative protein atoms (such as $\mathrm{O}, \mathrm{N}$, and $\mathrm{S}$ ), in view of field-induced water-dipole (re-) orientations breaking "prematurely" these types of hydrogen bonds (usually with rather prompt subsequent re-formation).

Naturally, considerations of aggregate behaviour of the number, and especially the lifetimes, of the protein-water hydrogen-bond network, in a global sense, obscure intriguing behaviour of individual residues and the solvation-layer "bound" water molecules in their locale that only a residueby-residue analysis of hydrogen-bond kinetics can illuminate.
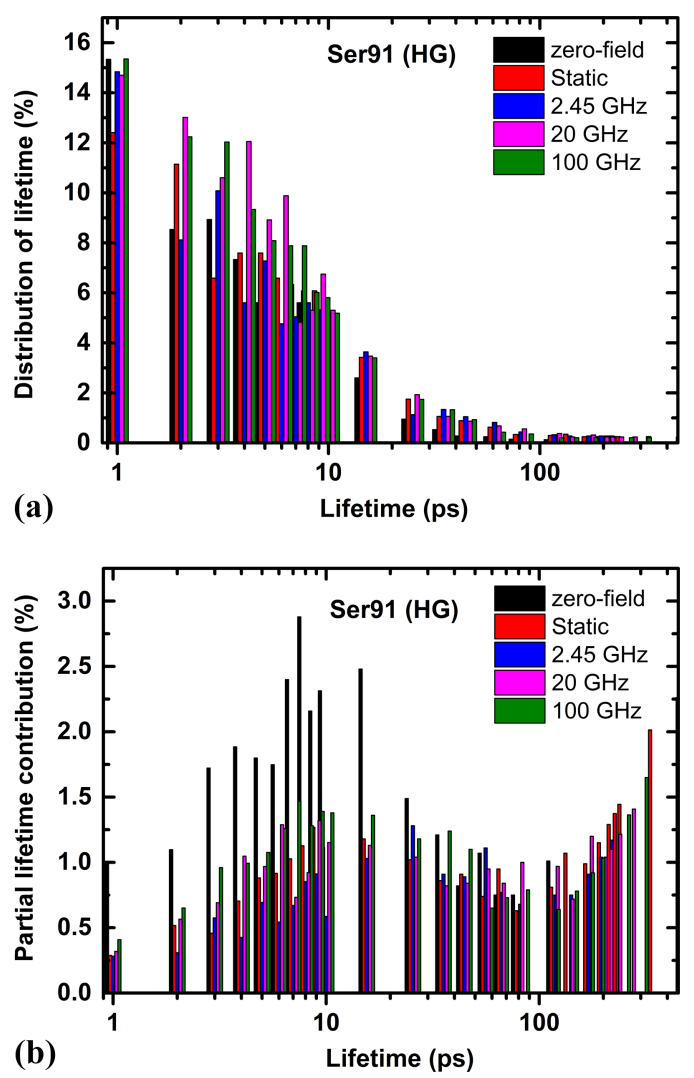

FIG. 10. Plots showing distribution of lifetime (a) and partial lifetime contribution (b) of the lifetime for the hydrogen bond formed by a Ser91 OPLS-HG-type proton with a water oxygen atom, at $300 \mathrm{~K}$.
Indeed, a panoply of residue-specific in-field perturbations comes to the fore, highlighting a rich tapestry of behaviour. To this end, histograms are provided in Figs. 6-10 showing the distribution of lifetime and partial-lifetime contribution of lifetime for hydrogen bonds formed by a selection of protein residues of proton sites to a water-molecule oxygen atom at $300 \mathrm{~K}$. This temperature was chosen in view of avoiding any supercooling of the hydration- (and bulk-) water, and proton sites were selected in view of removing any "artefacts" in hydrogen-bond-arrangement quickening by water-dipole orientations breaking "prematurely" hydrogen bonds between water protons and electronegative protein atoms (such as $\mathrm{O}, \mathrm{N}$, and $\mathrm{S}$ ). The distribution is seen to be influenced considerably by the presence of external electric fields, owing in large part to the net-charge and dipole-magnitude status of the residues in question. ${ }^{12}$ For instance, the acceleration in rearrangement kinetics is especially marked for Glu35 and Trp62, with the static field being most perturbative for a residue with negative charge- - owing to the local translational motion tending against (i.e., attempted, by-and-large unsuccessfully, in the opposite direction to) that of the gross electrophoretic motion of the (positively charged) HEWL as a whole. In contrast, the e/m fields exhibit the most dramatic shift in the case of substantially dipolar residues, in terms of inducing attempted (and typically frustrated) continuous local-residue rotational motion, thereby accelerating residue-water hydrogen-bond rearrangement kinetics.

\section{CONCLUSIONS}

It was found that the translational self-diffusivity of HEWL and its hydration-water layer are increased substantially in static electric fields at $300 \mathrm{~K}$, primarily due to induced electrophoresis, with the greater viscosity of supercooled bulk water $200 \mathrm{~K}$ serving to inhibit electrophoresis in relative terms. The water-protein hydrogen-bond-networkrearrangement kinetics can also undergo rather striking accelerations, primarily due to the enhancement of larger-amplitude local translational and rotational motion by charged and dipolar residues, which serves to promote hydrogen-bond breakage and re-formation kinetics. These external-field effects are particularly evident at $200 \mathrm{~K}$, where they serve to induce proteinand solvation-layer-response effects redolent of dynamical transition at a lower temperature $(\sim 200 \mathrm{~K})$ vis-à-vis the zerofield case $(\sim 220 \mathrm{~K})$. This de facto inducement of lowertemperature dynamical crossover by external electric fields signals their potential exploitation as an agent for activating, and indeed regulating, biological activity, especially at low temperatures.

\section{SUPPLEMENTARY MATERIAL}

See supplementary material for mean-square displacements of protein and water, alongside further residue-byresidue protein-water hydrogen-bonding details.

\section{ACKNOWLEDGMENTS}

All authors thank Science Foundation Ireland (Grant No. 15/ERC/I3142). 
${ }^{1}$ D. I. de Pomerai, B. Smith, A. Dawe, K. North, T. Smith, D. B. Archer, I. R. Duce, D. Jones, and P. M. Candido, FEBS Lett. 543(1-3), 93 (2003); M. Porcelli, G. Cacciapuoti, S. Fusco, R. Massa, G. dAmbrosio, C. Bertoldo, M. DeRosa, and V. Zappia, ibid. 402(2-3), 102 (1997).

${ }^{2}$ L. S. Taylor, Bioelectromagnetics 2(3), 259 (1981).

${ }^{3}$ M. H. Repacholi, Toxicol. Lett. 120(1-3), 323 (2001).

${ }^{4}$ J. Thuéry and E. H. Grant, Microwaves : Industrial, Scientific, and Medical Applications (Artech House, Boston, 1992).

${ }^{5}$ A. M. Phelan, C. F. Neubauer, R. Timm, J. Neirenberg, and D. G. Lange, Radiat. Res. 137(1), 52 (1994).

${ }^{6}$ H. Bohr and J. Bohr, Phys. Rev. E 61, 4310 (2000).

${ }^{7}$ R. Weissenborn, K. Diederichs, W. Welte, G. Maret, and T. Gisler, Acta Crystallogr., Sect. D: Biol. Crystallogr. 61, 163 (2005).

${ }^{8}$ B. Réjasse, T. Besson, M. D. Legoy, and S. Lamare, Org. Biomol. Chem. 4, 3703 (2006).

${ }^{9}$ J. E. Shea and C. L. Brooks, Annu. Rev. Phys. Chem. 52, 499 (2001).

${ }^{10}$ N. J. English and C. J. Waldron, Phys. Chem. Chem. Phys. 17, 12407 (2015).

${ }^{11}$ N. J. English and D. A. Mooney, J. Chem. Phys. 126(9), 091105 (2007).

${ }^{12}$ N. J. English, G. Y. Solomentsev, and P. O’Brien, J. Chem. Phys. 131, 035106 (2009).

${ }^{13}$ G. Y. Solomentsev, N. J. English, and D. Mooney, J. Chem. Phys. 133, 235102 (2010).

${ }^{14}$ J. A. Rupley and G. Careri, Advances in Protein Chemistry, edited by C. B. Anfinsen, J. T. Edsall, F. M. Richards and D. S. Eisenberg (Academic Press, 1991), Vol. 41, pp. 37-172.

${ }^{15}$ B. F. Rasmussen, A. M. Stock, D. Ringe, and G. A. Petsko, Nature 357, 423 (1992).

${ }^{16}$ A. Lee and W. A. Joshua, Nature 411, 501 (2001).

${ }^{17}$ P. W. Fenimore, H. Frauenfelder, B. H. McMahon, and R. D. Young, Proc. Natl. Acad. Sci. U. S. A. 101, 14408 (2004).

${ }^{18}$ F. G. Parak, Curr. Opin. Struct. Biol. 13, 552 (2003).

${ }^{19}$ M. Ferrand, A. J. Dianoux, W. Petry, and G. Zaccai, Proc. Natl. Acad. Sci. U. S. A. 90, 9668 (1993).

${ }^{20}$ W. Doster, S. Cusak, and W. Petry, Nature 337, 754 (1989).

${ }^{21}$ S. H. Chen, L. Liu, E. Fratini, P. Baglioni, A. Faraone, and E. Momontov, Proc. Natl. Acad. Sci. U. S. A. 103, 9012 (2006).

${ }^{22}$ G. Schiro, Y. Fichou, F.-X. Gallat, K. Wood, F. Gabel, M. Moulin, M. Hartlein, M. Heyden, J.-P. Colletier, A. Orecchini, A. Paciaroni, J. Wuttke, D. Tobias, and M. Weik, Nat. Commun. 6, 6490 (2015).

${ }^{23}$ H. Frauenfelder, G. Chen, J. Berendzen, P. W. Fenimore, H. Jannson, B. H. Mcmahon, I. R. Stroe, J. Swenson, and R. D. Young, Proc. Natl. Acad. Sci. U. S. A. 106, 5129 (2009).

${ }^{24}$ S. Khodadadi, S. Pawlus, J. H. Row, V. G. Sakai, E. Mamontov, and A. P. Sokolov, J. Chem. Phys. 128, 195106 (2008).

${ }^{25}$ S. Magazu, F. Migliardo, and A. Benedetto, Rev. Sci. Instrum. 82, 105115 (2011).

${ }^{26}$ S. Magazu, F. Migliardo, and A. Benedetto, J. Phys. Chem. B 115, 7736 (2011).

${ }^{27}$ T. Becker, J. A. Hayward, J. L. Finney, R. M. Daniel, and J. C. Smith, Biophys. J. 87, 1436 (2004).

${ }^{28}$ R. D. Young, H. Frauenfelder, and P. Fenimore, Phys. Rev. Lett. 107, 158102 (2011).

${ }^{29}$ B. Bagchi, "Hydration of proteins," in Water in Biological and Chemical Processes: From Structure and Dynamics to Function (Cambridge University Press, 2013), pp. 117-134.
${ }^{30}$ N. Galamba, J. Phys. Chem. B 118, 4169 (2014).

${ }^{31}$ J. T. Titantah and M. Karttunen, J. Am. Chem. Soc. 134, 9362-9368 (2012).

${ }^{32}$ D. A. Zichi and P. J. Rossky, J. Chem. Phys. 84, 2814 (1986).

${ }^{33}$ M. Jahn and S. Gekle, Phys. Rev. E 92, 052130-1-052130-10 (2015).

${ }^{34}$ M. Heyden, D. J. Tobias, and D. V. Matyushov, J. Chem. Phys. 137, 235103-1-235103-9 (2012).

${ }^{35}$ M. Ahmad, W. Gu, T. Geyer, and V. Helms, Nat. Commun. 2, 261 (2011).

${ }^{36}$ S. Gekle and R. R. Netz, J. Chem. Phys. 137, 104704 (2012).

${ }^{37}$ S. Gekle and R. R. Netz, J. Phys. Chem. B 118, 4963 (2014).

${ }^{38} \mathrm{~N}$. Todorova, A. Bentvelzen, N. J. English, and I. Yarovsky, J. Chem. Phys. 144, 085101 (2016).

${ }^{39}$ D. V. D. Spoel, E. Lindahl, B. Hess, G. Groenhof, A. E. Mark, and H. J. C. Berendsen, "GROMACS: Fast, flexible, and free," J. Comput. Chem. 26, 1701 (2005)

${ }^{40}$ W. L. Jorgensen, D. S. Maxwell, and J. Tirado-Rives, J. Am. Chem. Soc. 118, 11225-11236 (1996).

${ }^{41}$ G. A. Kaminski, R. A. Friesner, J. Tirado-Rives, and W. L. Jorgensen, J. Phys. Chem. B 105, 6474 (2001).

${ }^{42}$ H. J. C. Berendsen, J. R. Grigera, and T. P. Straatsma, J. Phys. Chem. 91, 6269 (1987).

${ }^{43}$ M. Ramanadham, L. C. Sieker, and L. H. Jensen, Acta Crystallogr., Sect. B: Struct. Sci. 46, 63 (1990).

${ }^{44}$ B. Hess, H. Bekker, H. J. C. Berendsen, and J. G. E. M. Fraaije, J. Computational Chem. 18, 1463 (1997).

${ }^{45}$ U. Essmann, L. Perera, M. L. Berkowitz, T. Darden, H. Lee, and L. G. Pedersen, J. Chem. Phys. 103, 8577 (1995).

${ }^{46}$ N. J. English and J. M. D. MacElroy, J. Chem. Phys. 118(4), 1589 (2003).

${ }^{47}$ N. J. English and J. M. D. MacElroy, J. Chem. Phys. 119(22), 11806 (2003).

${ }^{48}$ M. N. Afsar and J. B. Hasted, J. Opt. Soc. Am. 67(7), 902 (1977); H. R. Zelsmann, J. Mol. Struct. 350(2), 95 (1995).

${ }^{49}$ F. Calvo and P. Dugourd, Biophys. J. 95(1), 18 (2008).

${ }^{50}$ J. D. Durrant and J. A. McCammon, J. Mol. Graphics Modell. 31, 5 (2011).

${ }^{51}$ S. Ebbinghaus, S. J. Kim, M. Heyden, X. Yu, U. Heugen, M. Greubele, D. M. Leitner, and M. Havenith, Proc. Natl. Acad. Sci. U. S. A. 104, 20749 (2007).

${ }^{52}$ G. F. Voronoi, J. Reine Angew. Math. 134, 198 (1908).

${ }^{53}$ B. Halle and M. Davidovic, Proc. Natl. Acad. Sci. U. S. A. 100, 12135 (2003).

${ }^{54}$ M. Holz, S. R. Heil, and A. Sacco, Phys. Chem. Chem. Phys. 2, 4740 (2000).

${ }^{55}$ R. Mills, J. Phys. Chem. 77, 685 (1973).

${ }^{56}$ A. J. Easteal, W. E. Price, and L. A. Woolf, J. Chem. Soc., Faraday Trans. 1 85, 1091 (1989)

${ }^{57}$ S. B. Dubin, N. A. Clark, and G. B. Benedek, J. Chem. Phys. 54, 5158 (1971).

${ }^{58}$ N. J. English and D. A. Mooney, Phys. Chem. Chem. Phys. 11, 9370 (2009).

${ }^{59}$ N. J. English, D. A. Mooney, and S. W. O’Brien, J. Mol. Liq. 157, 163 (2010)

${ }^{60}$ N. J. English, P. G. Kusalik, and S. A. Woods, J. Chem. Phys. 136, 094508 (2012)

${ }^{61}$ N. J. English, Mol. Phys. 104, 243 (2006).

${ }^{62}$ R. Reale, N. J. English, P. Marracino, M. Liberti, and F. Apollonio, Chem. Phys. Lett. 582, 60 (2013).

${ }^{63}$ R. Reale, N. J. English, P. Marracino, M. Liberti, and F. Apollonio, Mol. Phys. 122, 1870 (2014). 